\title{
Self-Controlled Feedback for a Complex Motor Task
}

\author{
Roland Sigrist \\ Georg Rauter \\ Robert Riener \\ Sensory Motor Systems Lab, ETH Zurich \& \\ Spinal Cord Injury Center, University Hospital Balgrist, Zurich, Switzerland \\ [roland.sigrist / georg.rauter / peter.wolf / robert.riener] @mavt.ethz.ch
}

\begin{abstract}
Self-controlled augmented feedback enhances learning of simple motor tasks. Thereby, learners tend to request feedback after trials that were rated as good by themselves. Feedback after good trials promotes positive reinforcement, which enhances motor learning. The goal of this study was to investigate when naïve learners request terminal visual feedback in a complex motor task, as conclusions drawn on simple tasks can hardly be transferred to complex tasks. Indeed, seven of nine learners stated to have intended to request feedback predominantly after good trials, but in contrast to their intention, kinematic analysis showed that feedback was rather requested randomly $(23 \%$ after good, $44 \%$ after intermediate, $33 \%$ after bad trials). Moreover, requesting feedback after good trials did not correlate with learning success. It seems that self-estimation of performance in complex tasks is challenging. As a consequence, learners might have focused on certain movement aspects rather than on the overall movement. Further studies should assess the current focus of the learner in detail to gain more insight in self-estimation capabilities during complex motor task learning.
\end{abstract}

\section{Introduction}

To facilitate intrinsic feedback and to develop a robust internal movement representation, the guidance hypothesis suggests giving feedback not on every trial. With a lower feedback frequency, reliance on the feedback is prevented, and thus, motor tasks can be successfully executed even when feedback is withdrawn [1]. The guidance hypothesis seems to be valid for simple motor tasks, but not necessarily for complex motor tasks. For complex tasks, especially in early learning stages, it might be helpful to provide feedback often to prevent excessive demand [2]. Thereby, a main challenge is to find an optimal feedback frequency for certain motor tasks at specific skill levels.
Different adaptations of feedback frequency to the increasing skill level have been proposed. Fading feedback reduces the feedback frequency over time, and has been shown to be effective [3]. However, the optimal fading rate is commonly unknown. Feedback reduction follows a rigid schedule and might, therefore, not be optimal for each individual. Bandwidth feedback is provided if the movement error exceeds (or is within) a certain threshold. Thereby, setting the error threshold is not trivial [4]. Self-controlled feedback allows the learner to choose the trials about which feedback should be provided. Advantages of selfcontrolled feedback are seen in the adaptation to the learner's needs, in that it allows a focus on the current aspect the learner wants to correct, in the promotion of deeper information processing, and in the involvement of the learner in the learning process resulting in an increased motivation [5].

Self-controlled feedback has been proven to be more effective than externally imposed feedback in ball throwing $[6,7]$, timing tasks $[8,9]$, and a motor perception task [10]. However, self-controlled feedback per se cannot be the only reason for better learning, as Chiviacowsky and Wulf [9] have shown: Participants that had to decide prior to the trial if they want to receive terminal feedback were outperformed by participants that could decide after the trial. It seems that feedback is most effective if it is provided after good trials, due to enhanced motivation and positive reinforcement to repeat good trials [11]. Indeed, selfcontrolled terminal feedback tends to be requested after learners' believed that they performed well $[8,9]$. Currently, studies have rarely reported on complex motor learning enhanced by self-controlled feedback. In particular, it remains unclear if learners of complex tasks request feedback mainly after good trials.

Therefore, the goal of the present work was to evaluate when naïve learners request terminal visual feedback in a complex motor task. It was hypothesized that learning was facilitated when learners request feedback mainly after good trials than after trials of intermediate or bad performance.

This is an Open Access article distributed under the terms of the Creative Commons Attribution-Noncommercial License 3.0, which permits unrestricted use, distribution, and reproduction in any noncommercial medium, provided the original work is properly cited. 


\section{Method}

\subsection{Participants}

Nine healthy participants ( 3 females, 6 males, 24 to 30 years, mean age 27.4 years) took part in the study. They declared themselves to be non-rowers and to have normal vision. They signed an agreement that followed the guidelines of the local ethics commission, which had approved the experiment.

\subsection{Apparatus and task}

During the experiment, the participant was seated in a real but trimmed rowing boat, set up in the middle of a CAVE (Cave Automated Virtual Environment). Reflective markers were attached to a trimmed sweep rowing oar to track the movement with an optoelectrical motion tracking system (Qualisys, Goteborg, Sweden). The kinematic data was used for later analysis and to render a virtual elongation of the real oar in real-time (update rate: $\sim 62.5 \mathrm{~Hz}$ ) on a $4.44 \mathrm{~m} \times$ $3.33 \mathrm{~m}$ screen on the right hand side of the participant (Fig. 1).

The participant moved the sweep rowing oar with both hands and arms, but without moving the legs. During the whole study, the participant only felt the inertia of the oar moving freely through the air. Water resistance was not simulated.

The rowing-type reference oar movement included three oar angles (horizontal range of outer hand movement: $25^{\circ} \approx 0.50 \mathrm{~m}$; vertical range: $13^{\circ} \approx 0.26 \mathrm{~m}$; blade rotation at two orientations from steep to flat and vice versa: $\left.90^{\circ}\right)$. To increase task complexity, a rowing-type angular velocity profile was applied. The angular velocity in the pulling (drive) phase was double the velocity in the pushing (recovery) phase. One cycle lasted $6 \mathrm{~s}$.

\subsection{Procedure}

Each participant was invited on four consecutive days (days 1-4) and one week after day 4, i.e. day 11. On day 1, after a general instruction, participants familiarized themselves with the simulator. Therefore, they were asked to match during $120 \mathrm{~s}$ their brown oar blade with the blue reference oar blade performing a circular movement with constant velocity. The cycle had a similar range as the reference trajectory and lasted $8 \mathrm{~s}$. Thereafter, the reference movement was visually displayed for $60 \mathrm{~s}$. Participants were asked to memorize a blue oar moving on the reference trajectory. Thereafter, they had to perform that movement with their own oar in a $180 \mathrm{~s}$ baseline test

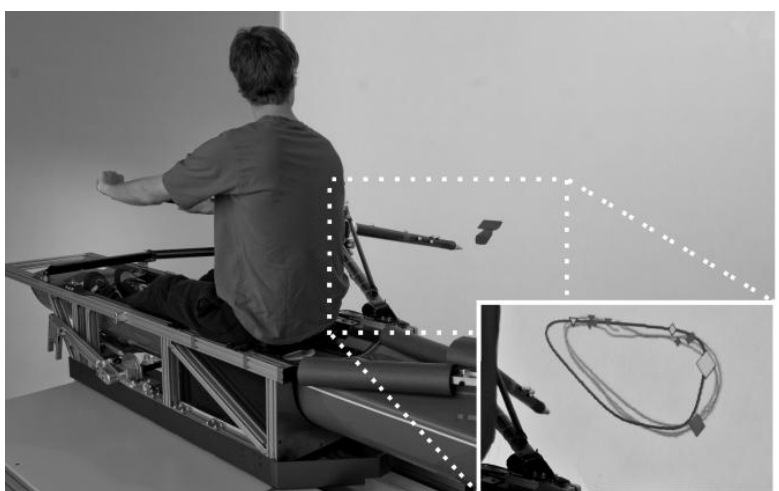

Fig. 1 Rowing boat with virtual oar blade and exemplary terminal feedback visualization

during which the reference oar was not displayed. After this baseline test, three trainings lasting on average $180 \mathrm{~s}$ were conducted, each followed by $30 \mathrm{~s}$ of catch trials. Days 2 and 3 started with a retention test, similar to the baseline test on day 1 . After the retention test, three trainings with self-controlled terminal visual feedback followed, as on day 1 . The durations of the three consecutive trainings varied $(150 \mathrm{~s}, 180 \mathrm{~s}$, or $210 \mathrm{~s})$ to avoid estimation when catch trials would start. Participants were verbally informed about the start of the catch trials, whereas the participants did not interrupt the movement. During catch trials, no feedback could be requested. On day 4 and 11 , a $180 \mathrm{~s}$ retention test was performed.

\subsection{Visual self-controlled feedback}

During the trainings, participants could request for feedback anytime they had completed at least three cycles, but not during catch trials. The feedback was displayed about $10 \mathrm{~s}$ after the request on the screen exactly at the position where the virtual oar blade was moved before. The feedback showed the reference trajectory together with the participant's performed trajectory of the last $18 \mathrm{~s}$, which is the period of 3 reference cycles. Blade rotations were indicated by the symbols (rotated to steep blade) and $\mathbf{\nabla}$ (rotated to flat blade) (Fig. 1, detail). Moreover, to provide feedback about the desired velocities, a replay of the own movement and the reference movement was shown. Reference and participant's blade were represented by abstract symbols, moving along the trajectories at the velocities as performed. Symbols changed every time the blades were rotated $(\bullet \leftrightarrow \mathbf{\nabla})$. The reference started its movement closest to the participant's starting replay position. After the replay, the participant could watch the trajectories for maximally $10 \mathrm{~s}$. The feedback visualization was programmed in Matlab (MathWorks, Natick, Massachusetts, USA). 


\subsection{Data analysis}

Kinematic data was analyzed in Matlab. Data was low pass filtered by fitting a smoothing spline to it. The first cycle and last two cycles of the baseline and retention runs were ignored as well as the first cycle of the training block, cycles shorter than $3 \mathrm{~s}$ or longer than $9 \mathrm{~s}$, and cycles with missing data caused by tracking errors.

Movements of the participants were compared to the reference movement. Data was cut into cycles at the minimal horizontal angle (release), as participants usually stopped the movement right afterwards. The horizontal and vertical angles were used for a spatial and temporal analysis of the trajectory [12]. In the algorithm, the reference and participant's trajectory are compared respecting their spatial and temporal properties to calculate spatial and temporal errors.

In baseline and retention tests, participants' trajectories were often shifted related to the absolute position of the reference. Relative errors were of more interest than shift errors. Thus, before calculating the relative errors, each trajectory was moved to the position where the spatial error was minimal. In trainings, when participants had an absolute reference trajectory given by the terminal feedback, the absolute spatial and temporal error was used.

To calculate timing errors of blade rotations, each completed cycle was taken as $100 \%$. The difference between the participants' blade rotations and the one of the reference cycle were calculated in percent.

Training runs were divided into blocks. A block ended with the feedback request of the participant. Cycles were assigned to "good" in respect of an error variable, i.e. spatial, temporal, and timing error of blade rotation, if the error was in lower quartile of all the cycles of the block, to "bad" if it was in the upper quartile, and to "intermediate" if it was in between.

For each error variable, the relative improvement from baseline to the retention tests on day 4 and 11 was calculated. It was proven if the improvement was correlated with the percentage of taking feedback after good, intermediate, or bad cycles (Pearson, $\mathrm{p}=.05$ ).

To asses learning, statistical analysis was done with the data of the baseline and retention tests by a Friedman test with post-hoc Bonferroni correction $(\mathrm{p}=$ $.05)$ by multiple comparison.

\section{Results}

Significant differences $(p<.05)$ from baseline to each of the retention tests on day 2, 3, 4, and 11 were found for the spatial error. No significant differences were found for the temporal error between baseline and

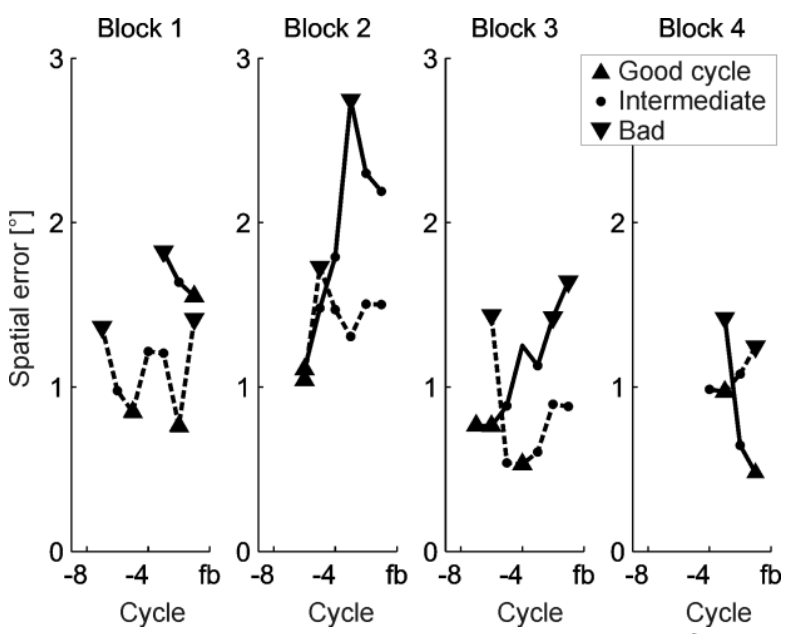

Fig. 2 Mean spatial error per cycle of two exemplary participants (solid \& dashed), day 2 , training 3 . fb: feedback request

any retention test. For timing error of blade rotation, the baseline test differed significantly to the retention tests of day 3, 4, and 11, but not of day 2 . Retention tests did not differ significantly from each other for all three error variables, i.e. spatial, temporal, and blade rotation error. In general, errors decreased from day 1 to day 4, and slightly increased from day 4 to 11 .

Concerning the spatial error, feedback was taken after a cycle of good performance in $20 \%$ (mean of all participants), after intermediate performance in $48 \%$, and after bad performance in $32 \%$. For the temporal error it was $26 \%$ after good, $41 \%$ after intermediate, and $33 \%$ after cycles of bad performance. For blade rotation it was $22 \%$ after good, $45 \%$ after intermediate, and $33 \%$ after bad timing. Visual inspection of all cycles in a block did not reveal any preferences of taking feedback after specific cycles (Fig. 2).Five participants reported to have tried to take feedback after good cycles, two more often after good but also after bad cycles, one after bad cycles, and one did not comment his strategy.

Only one out of 18 correlations was significant: Higher number of feedback requests after bad cycles correlated with performance increases from baseline to the retention test on day $11\left(\mathrm{R}^{2}=0.90\right)$. For all other correlations were not significant $\left(\mathrm{R}^{2}<0.44\right)$.

\section{Discussion and conclusions}

One goal of the study was to test the hypothesis that participants learn better if feedback is taken after good trials (cycles) rather than after bad trials. However, taking feedback after good, bad, or intermediate trials did not correlate with enhanced learning. Another goal was to elaborate when self-controlled feedback is 
mainly taken in complex motor task learning. It was hypothesized that feedback is requested after good trials, as done in simpler motor tasks $[6,7,8,9,10]$. The results of the present study suggest that feedback is not generally taken after good trials, but rather randomly. Interestingly, seven of nine participants reported to intend to take feedback dominantly after good trials.

One reason of the discrepancy of participants' reports and the kinematic analysis could be that selfestimation of the performance was too difficult for the task in this study. Thus, even though they wanted to take feedback after good trials, they were not able to estimate their performance correctly. Referring to a general definition of task complexity [2], the rowingtype movement was rather complex as it had several degrees of freedom, i.e. three oar angles and a complex velocity profile; and it could not be learned in a single training. The tasks had high demands on short-term memory, motor planning and control, and long-term memory of the multi-joint body-arm movement. The results show that participants' errors were significantly lower after one training day concerning the spatial error and after two days concerning timing of blade rotation. They could not significantly decrease the temporal error until the end of all trainings. This might be because in the terminal feedback in form of a replay, temporal errors were more difficult to perceive than spatial errors. Overall, the movement can be assumed to be rather complex.

Another reason can be, as many participants reported, that they focused on certain aspects of the movement rather than on the overall movement. Thus, indeed, they could have taken feedback after good performance regarding the specific aspect, but the calculated errors could still have been high, as they resulted from whole cycle analysis.

Moreover, some participants reported that not only the last, but the last two or three cycles prior to the request must have been good to decide to take feedback. A more detailed assessment of the participant's current strategy to take feedback is necessary to improve the analysis and gain more specific results.

\section{Outlook}

In complex tasks, self-estimation might be too difficult to detect good trials. However, self-estimation has been declared to be important for learning $[13,14]$. In further studies, it should be evaluated how task complexity affects self-estimation. Moreover, it should be investigated if externally imposed feedback after good trials is more effective than self-controlled feedback, which promotes self-estimation. Finally, it would be interesting to compare self-controlled terminal feedback to other types of feedback, e.g. concurrent feedback provided in different modalities, to figure out which type of feedback facilitates learning of complex motor tasks best.

\section{References}

[1] S. Salmoni. Knowledge of results and motor learning. a review and critical reappraisal. Psychol Bull, 95(3):355-386, 1984.

[2] G. Wulf and C. H. Shea. Principles derived from the study of simple skills do not generalize to complex skill learning. Psychon Bull Rev, 9(2):185-211, 2002.

[3] A. J. Kovacs and C. H. Shea. The learning of $90^{\circ}$ continuous relative phase with and without lissajous feedback: External and internally generated bimanual coordination. Acta Psychol, 136(3):311-320, 2011.

[4] D. C. Ribeiro, G. Sole, J. H. Abbott, and S. Milosavljevic. Extrinsic feedback and management of low back pain: A critical review of the literature. Man Ther, 16(3):231-239, 2011.

[5] G. Wulf. Self-controlled practice enhances motor learning: implications for physiotherapy. Physiotherapy, 93(2):96-101, 2007.

[6] C. M. Janelle, D. A. Barba, S. G. Frehlich, L. K. Tennant, and H. Cauraugh. Maximizing performance feedback effectiveness through videotape replay and a selfcontrolled learning environment. Res $Q$ Exerc Sport, 68(4):269-279, 1997.

[7] C. M. Janelle, J. Kim, and R. N. Singer. Subjectcontrolled performance feedback and learning of a closed motor skill. Percept Mot Skills, 81(2):627-634, 1995.

[8] S. Chiviacowsky and G. Wulf. Self-controlled feedback: does it enhance learning because performers get feedback when they need it? Res $Q$ Exerc Sport, 73(4):408415, 2002.

[9] S. Chiviacowsky and G. Wulf. Self-controlled feedback is effective if it is based on the learner's performance. Res Q Exerc Sport, 76(1):42-48, 2005.

[10] M. Huet, C. Camachon, L. Fernandez, D. M. Jacobs, and G. Montagne. Self-controlled concurrent feedback and the education of attention towards perceptual invariants. Hum Mov Sci, 28(4):450-467, 2009.

[11] S. Chiviacowsky and G. Wulf. Feedback after good trials enhances learning. Res Q Exerc Sport, 78:40-47, 2007.

[12] M.A. Giese and T. Poggio. Morphable models for the analysis and synthesis of complex motion patterns. International Journal of Computer Vision, 38(1):59-73, 2000.

[13] M.A. Guadagnoli and R.M. Kohl. Knowledge of results for motor learning: relationship between error estimation and knowledge of results frequency. J Mot Behav, 33(2):217-224, 2001.

[14] J. Liu and C. A. Wrisberg. The effect of knowledge of results delay and the subjective estimation of movement form on the acquisition and retention of a motor skill. Res $Q$ Exerc Sport, 68(2):145-151, 1997. 